\title{
The 2017 ACC/AHA Hypertension Guidelines: Should they have included proven nonpharmacological blood pressure- lowering strategies such as Transcendental Meditation?
}

Dear Editor,

We appreciate that the recent ACC/AHA High Blood Pressure Guidelines ${ }^{1}$ recommend nonpharmacologic interventions for prevention and treatment of hypertension. However, we believe that the guidelines made an error when distinguishing between (a) effective nonpharmacological interventions for lowering blood pressure and (b) other interventions that lacked strong evidence for their long-term BPlowering effect. The former included weight loss, healthy diet, reduced intake of dietary sodium, enhanced intake of dietary potassium, physical activity, and moderation in alcohol intake. The latter included behavioral therapies such as guided breathing, yoga, Transcendental Meditation, and biofeedback.

In particular, the review of studies of Transcendental Meditation for the treatment of high blood pressure which was completed in 2017 comprised only one reference, ${ }^{2}$ and that one reference was limited to studies or reviews completed prior to 2004. Therefore, the 2017 BP Guidelines did not include what is now 12 randomized controlled trials and several systematic reviews and meta-analyses on Transcendental Meditation and blood pressure ${ }^{3-6}$ that were published in the 13-year gap between 2004 and 2017. This is hardly an up-to-date systematic review in the current ACC/AHA guidelines.

Second, the 2017 BP guidelines did not include the scientific statement from the American Heart Association on alternative methods for lowering BP.7 This statement concluded that Transcendental Meditation may be effective in reducing high blood pressure and that it was the only meditation technique with sufficient data at the time to merit that recommendation. The reductions in SBP and DBP elicited by Transcendental Meditation were statistically and clinically significant (approx. $-5 /-3 \mathrm{~mm} \mathrm{Hg}$, respectively). Furthermore, published randomized controlled trials have reported that this meditation practice, when used in conjunction with usual medical care, is associated with reductions in mortality ${ }^{8}$ and in the composite of all-cause mortality, myocardial infarction, and stroke. ${ }^{9}$

In light of these more recent findings, we suggest that the AHA committee update its review and recommendations on nonpharmacologic interventions for blood pressure, particularly in regard to the Transcendental Meditation technique. Patients with high blood pressure should be made aware that there is another evidence-based option for the prevention and treatment of high blood pressure that may help to mitigate the need for drug therapy.

\section{CONFLICT OF INTEREST}

The authors report no conflicts of interest to disclose. Robert H. Schneider MD, FACC ${ }^{1}$ Jeremy Z. Fields $\mathrm{PhD}^{2}$ Robert D. Brook MD ${ }^{3}$

${ }^{1}$ College of Integrative Medicine, Maharishi University of Management, Fairfield, lowa Email:RSchneider@mum.edu

${ }^{2}$ CATX, Inc, Freeport, Maine

${ }^{3}$ University of Michigan Medical School, Ann Arbor, Michigan Email:RSchneider@mum.edu

\section{REFERENCES}

1. Whelton PK, Carey RM, Aronow WS, et al. 2017 ACC/AHA/AAPA/ ABC/ACPM/AGS/APhA/ASH/ASPC/NMA/PCNA Guideline for the Prevention, Detection, Evaluation, and Management of High Blood Pressure in Adults: A Report of the American College of Cardiology/ American Heart Association Task Force on Clinical Practice Guidelines. Hypertension. 2018;71:1269-1324.

2. Canter PH, Ernst E. Insufficient evidence to conclude whether or not Transcendental Meditation decreases blood pressure: results of a systematic review of randomized clinical trials. J Hypertens. 2004;22(11):2049-2054.

3. Bai Z, Chang J, Chen C, Li P, Yang K, Chi I. Investigating the effect of transcendental meditation on blood pressure: a systematic review and meta-analysis. J Hum Hypertens. 2015;29:653-662.

4. Rainforth M, Schneider R, Nidich S, King C, Salerno J, Anderson J. Stress reduction programs in patients with elevated blood pressure: a systematic review and meta-analysis. Curr Hypertens Rep. 2007;9(6):520-528.

5. Anderson J, Liu C, Kryscio R. Blood pressure response to transcendental meditation: a meta-analysis. Am J Hypertens. 2008;21(3):310-316.

6. Ooi SL, Giovino M, Pak SC. Transcendental meditation for lowering blood pressure: an overview of systematic reviews and meta-analyses. Complement Ther Med. 2017;34:26-34.

7. Brook RD, Appel LJ, Rubenfire M, et al. Beyond medications and diet: alternative approaches to lowering blood pressure: a scientific statement from the American Heart Association. Hypertension. 2013;61:1360-1383.

8. Schneider RH, Alexander CN, Staggers F, et al. Long-term effects of stress reduction on mortality in persons $>55$ years of age with systemic hypertension. Am J Cardiol. 2005;95(9):1060-1064.

9. Schneider R, Grim C, Rainforth M, et al. Stress reduction in the secondary prevention of cardiovascular disease: randomized controlled trial of Transcendental Meditation and health education in Blacks. Circulation. 2012;5:750-758. 